\title{
CircRNA circVEGFC is Highly Expressed in Gestational Diabetes Mellitus (GDM) and It is Correlated with Multiple Adverse Events
}

\section{Wenjing She \\ Tao Li \\ Yan Liu \\ Xinru Liu}

West China Second Hospital of Sichuan University/Key Laboratory of Birth Defects and Related Diseases of Women and Children (Sichuan University), Ministry of Education, Chengdu City, 6I004I, People's Republic of China
Correspondence: Xinru Liu

West China Second Hospital of Sichuan University/Key Laboratory of Birth Defects and Related Diseases of Women and Children (Sichuan University), Ministry of Education, No. 20, Section, 3. Renmin South Road, Wuhou District, Chengdu City, 61004I, People's Republic of China

Tel +86-028-85503067

Email xinruliudisease@।63.com
Background: Circular RNA vascular endothelial growth factor $\mathrm{C}$ (circVEGFC) is a novel regulator of glucose metabolism, while its role in gestational diabetes mellitus (GDM) is unclear. This study aimed to detect the expression of circVEGFC in GDM and explore its clinical values. Methods: This study enrolled 220 pregnant women (gestational age less than 5 weeks) with normal blood glucose level on the day of admission. The expression of circVEGFC in plasma samples of these participants was determined by RT-qPCR. The participants were divided into high and low circVEGFC level groups with the median expression level of plasma circVEGFC as the cutoff value. The development of GDM was monitored until delivery. Adverse events were also monitored.

Results: Compared to low circVEGFC level group, GDM-free curve analysis revealed significantly higher incidence of GDM in high circVEGFC level group. In addition, plasma expression levels of circVEGFC were also higher in GDM patients than that in non-GDM patients on the day of admission and at 1 month before and after delivery. ROC curve analysis revealed that high expression levels of circVEGFC on the day of admission showed higher sensitivity and specificity in the early diagnosis of GDM. Moreover, high circVEGFC level group showed higher incidence rates of fetal malformation and hypertension.

Conclusion: Therefore, circVEGFC is highly expressed in GDM, and it is correlated with multiple adverse events.

Keywords: circVEGFC, gestational diabetes mellitus, adverse events

\section{Introduction}

In many cases, pregnant women may not produce enough insulin, resulting in the development of gestational diabetes mellitus (GDM). ${ }^{1}$ GDM is a serious complication of pregnancy caused by the dysfunction of pancreatic $\beta$ cells, which are responsible for the production, storage and secretion of insulin. ${ }^{2,3}$ It was estimated that more than $12 \%$ of pregnant women will develop GDM, and the incidence increases with aging. ${ }^{4}$ Without proper treatment, GDM may cause adverse events to both mother and the fetus. ${ }^{5,6}$ Exercise, proper hydration, stress and sleep management and diet can be applied to control blood glucose in GDM patients. In severe cases, insulin injection can be performed. ${ }^{7}$ However, adverse events may inevitably occur. Therefore, novel approaches are needed to improve patients' conditions.

At present, GDM is usually screened between 24 and 28 weeks of gestational age. ${ }^{8}$ However, pregnant women with high risk may develop GDM at early gestational age and the delayed diagnosis is the main cause of most adverse events. ${ }^{9}$ Therefore, the 
development of early diagnostic predictor is still the key to improve the conditions of GDM patients and prevent adverse complications. ${ }^{10,11}$ Circular RNAs (circRNAs) are covalently linked long non-coding RNAs (lncRNAs) by back-splicing of exons. ${ }^{12}$ CircRNAs do not encode proteins and regulate human diseases, such as diabetes, mainly by regulating the expression of targeted genes. ${ }^{12}$ In effect, some differentially expressed circRNAs may serve as potential biomarkers for human diseases including GDM. ${ }^{12-14}$ Circular RNA vascular endothelial growth factor $\mathrm{C}$ (circVEGFC) is novel regulator of glucose metabolism. ${ }^{15}$ CircVEGFC can promote high glucose-induced injuries of vascular endothelial cells by interacting with the miR-338-3p/HIF-1 $\alpha /$ VEGFA axis, ${ }^{15}$ while its role in GDM is unknown. This study aimed to detect the expression of circVEGFC in GDM and explore its clinical values.

\section{Materials and Methods}

\section{Participants and Follow-Up}

The present study enrolled a total of 220 pregnant women (gestational age less than 5 weeks) who were admitted at West China Second Hospital of Sichuan University/Key Laboratory of Birth Defects and Related Diseases of Women and Children (Sichuan University), Ministry of Education from May 2018 to May 2021. During this time period, 1244 women in early pregnancy (the number of all pregnant women enrolled was more than 10 times higher) were admitted to this hospital, but only 248 out of them agreed to participate in this study. Moreover, 28 out of the 248 women were excluded due to the existence of certain clinical disorders. Blood samples were collected from all participants to exclude high blood glucose. Participants with a history of any types of diabetes were excluded from this study. This study also excluded participants with severe diseases, such as cancer, metabolic disorders and chronic infections. Patients were monitored until one month after delivery. A blood sugar level higher than 190 $\mathrm{mg} / \mathrm{dL}$ prior to delivery was used as the criteria to diagnose GDM. During follow-up, adverse events were also monitored and recorded. The Ethics Committee of West China Second Hospital of Sichuan University/Key Laboratory of Birth Defects and Related Diseases of Women and Children (Sichuan University), Ministry of Education approved this study. This study was conducted in accordance with the Declaration of Helsinki. All patients signed the informed consent.

\section{Preparation of RNA Samples}

Total RNAs were extracted from both in vitro cultivated cells and tissues from patients using GenElute ${ }^{\mathrm{TM}}$ Mammalian Total RNA Miniprep Kit. Total RNA samples were separated through centrifugation using silica membranes. After that, DNase I was used to remove genomic DNA in the RNA samples. RNA concentration and integrity were determined using Bioanalyzer.

\section{Preparation of cDNA Samples and qPCRs}

A total of $1 \mu \mathrm{g}$ total RNAs were used to synthesize cDNA samples using iScript ${ }^{\mathrm{TM}}$ cDNA Synthesis Kit (Bio-Rad). The prepared cDNA samples were used as template to perform qPCRs to determine the expression levels of circVEGFC with $18 \mathrm{~S}$ rRNA as the internal control. $\mathrm{Ct}$ values of circVEGFC were normalized to $18 \mathrm{~S}$ rRNA through the method of delta $\mathrm{Ct}$.

\section{Statistical Analysis}

Comparisons between two groups were performed using Student's $t$-test. With the median expression level of circVEGFC as the cutoff value, the 220 participants were divided into high and low circVEGFC level groups $(n=110)$. GDM-free curves were plotted for both groups and compared by Log rank test. Diagnostic value of the plasma expression levels of circVEGFC on the day of admission for GDM were analyzed with ROC curve analysis. Repeated measures ANOVA was applied to compare multiple time points in the same group. $P<0.05$ was statistically significant.

\section{Results}

\section{High Plasma Expression Levels of circVEGFC Predicted High Incidence of GDM}

To explore the predictive value of plasma circVEGFC on the day of admission for GDM, the 220 participants were

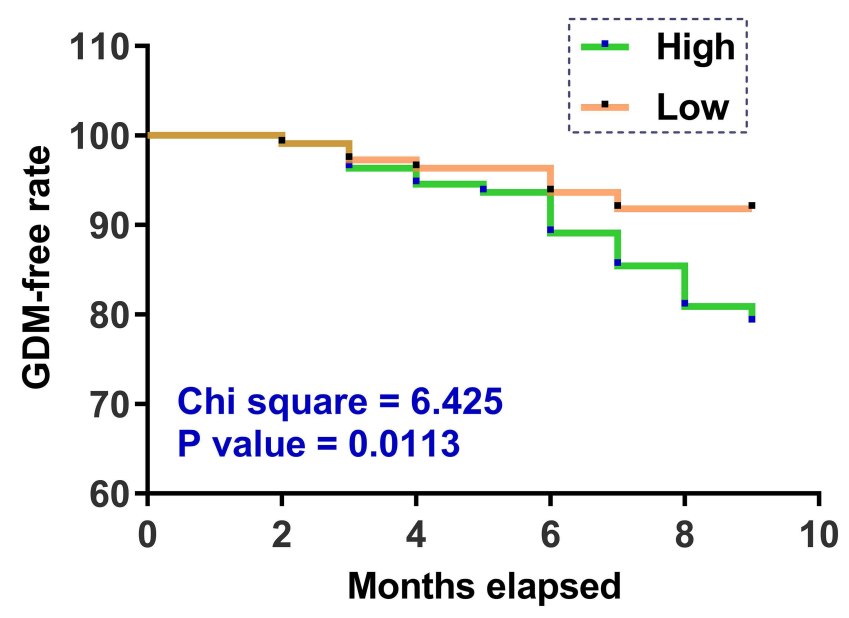

Figure I High plasma expression levels of circVEGFC predicted high incidence of GDM. To explore the predictive value of plasma circVEGFC on the day of admission for GDM, the 220 participants were divided into high and low circVEGFC level groups $(n=$ I10) with the median expression of plasma circVEGFC as the cutoff value. GDM-free curves were plotted for both groups and compared by Log rank test. 
divided into high and low circVEGFC level groups $(\mathrm{n}=$ 110 ) with the median expression level of circVEGFC as the cutoff value. GDM-free curves were plotted for both groups and compared by Log rank test. A total of 32 cases of GDM were diagnosed. Compared to low circVEGFC level group, GDM-free curve analysis revealed significantly higher incidence of GDM in high circVEGFC level group (Figure 1). Therefore, high plasma expression levels of circVEGFC predicted higher incidence of GDM.

\section{The Expression Levels of circVEGFC Were Higher in GDM Patients Than That in Non-GDM Patients at Multiple Time \\ Points}

According to the diagnosis of GDM, the 220 patients were divided into GDM ( $\mathrm{n}=32)$ and non-GDM groups $(\mathrm{n}=188)$.

Plasma expression levels of circVEGFC were compared

A

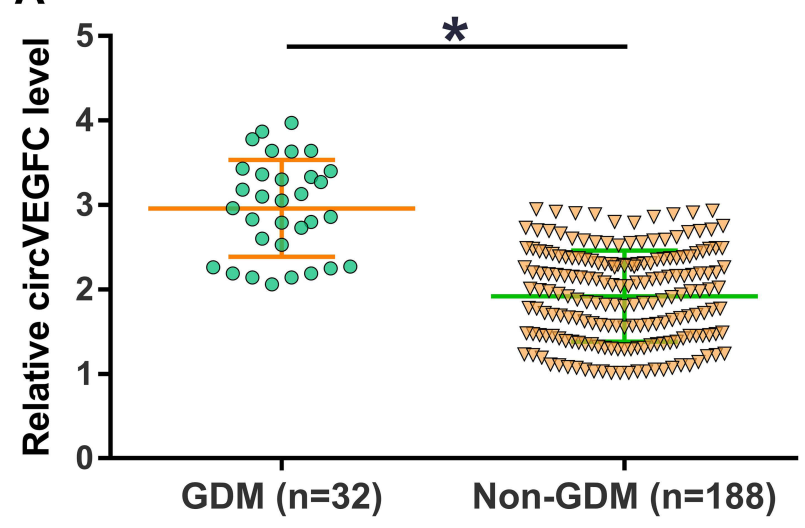

C

Admission

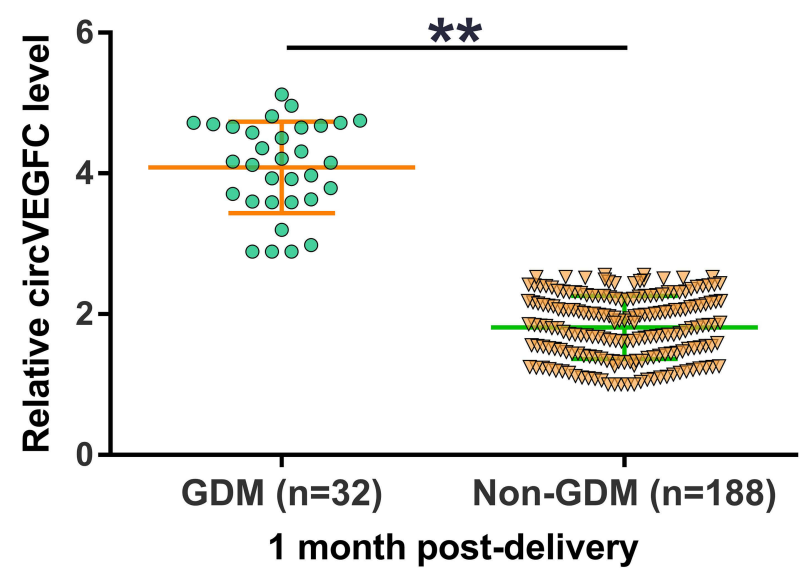

between these two groups at 3 time points, including the day of admission (Figure 2A), one month prior to delivery (Figure 2B) and 1 month after delivery (Figure 2C). At all 3 time points, plasma expression levels of circVEGFC were higher in GDM patients than that in non-GDM patients ( $p<$ 0.05 or $p<0.01$ ). Diabetes (type 2) was detected in all GDM patients at 6 and 12 weeks after delivery. Only 2 cases of diabetes were diagnosed at 6 weeks after delivery and only 1 case was diagnosed at 12 weeks after delivery.

\section{The Expression Levels of circVEGFC Were Increased in GDM Group, but Not in Non- GDM Group During the Follow-Up}

Plasma expression levels of circVEGFC were compared among the 3 time points, including the day of admission, one month prior to delivery and 1 month after delivery, in both GDM (Figure 3A) and non-GDM (Figure 3B) groups.

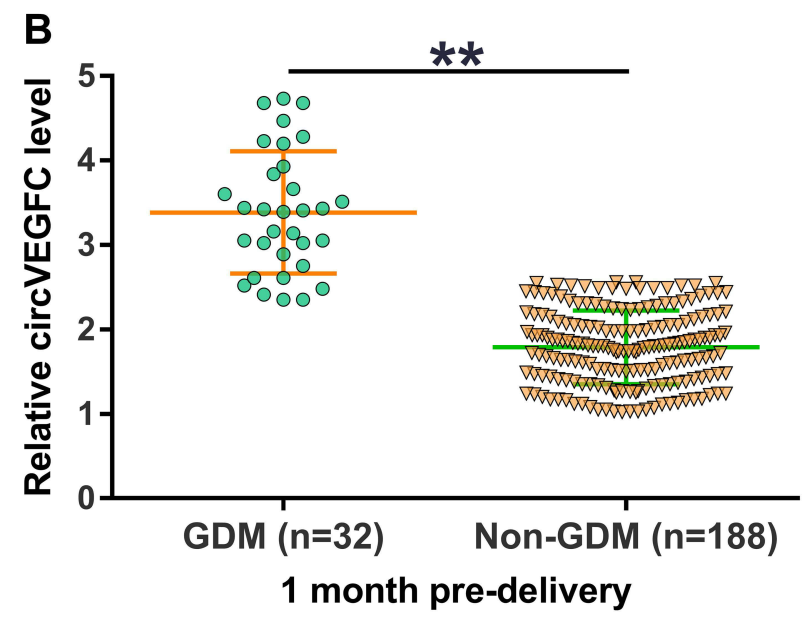

Figure 2 Plasms expression levels of circVEGFC were also higher in GDM patients than that in non-GDM patients at multiple time points. According to the diagnosis of GDM, the 220 patients were divided into GDM $(n=32)$ and non-GDM groups $(n=188)$. Plasma expression levels of circVEGFC were compared between two groups at three time points, including the day of admission $(\mathbf{A})$, one month prior to delivery $(\mathbf{B})$ and I month after delivery $(\mathbf{C})$. $*_{p}<0.05$; $*^{*} p<0.01$. 

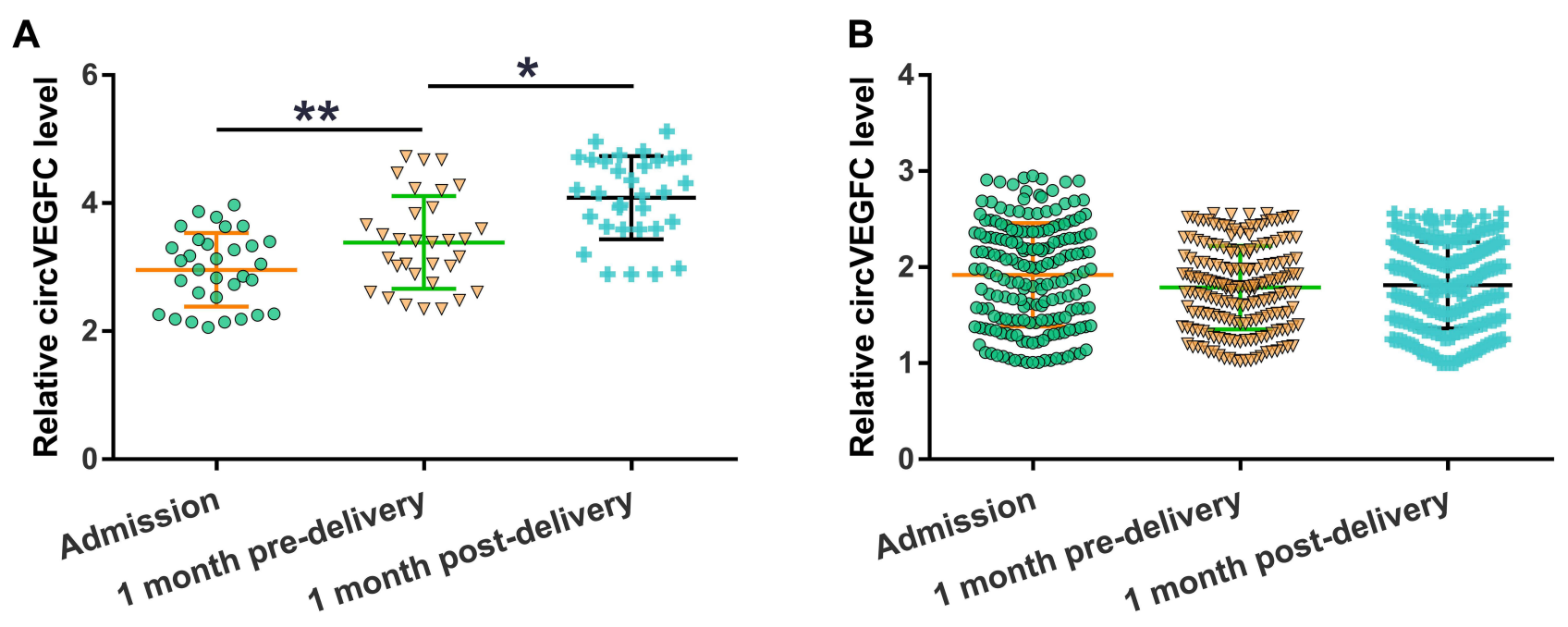

Figure 3 Plasma expression levels of circVEGFC increased in GDM group, but not in non-GDM group during follow-up. Plasma expression levels of circVEGFC were compared among three time points, including the day of admission, one month prior to delivery and I month after delivery, in both GDM (A) and non-GDM (B) groups. *p < $0.05 ; * p<0.01$.

It was observed that plasma expression levels of circVEGFC were significantly increased in GDM group (Figure 3A, $p<0.05$ or $p<0.01$ ), but not in non-GDM group (Figure 3B) during the follow-up.

\section{The Expression Levels of circVEGFC Provide High Sensitivity and Specificity in Early Diagnosis of GDM}

With GDM patients as true positive cases and non-GDM patients as true negative cases, the early diagnostic value of plasma expression levels of circVEGFC for GDM was analyzed by performing ROC curve analysis. High expression levels of circVEGFC on the day of admission showed higher sensitivity and specificity in the early diagnosis of GDM (Figure 4; area under the curve $=0.8927$ ).

\section{High Expression Levels of circVEGFC} Were Correlated with Fetal Malformation and Hypertension

Occurrence of multiple adverse events, including premature delivery, miscarriage, intrauterine distress, intrauterine death, intrauterine infection, fetal malformation, hypertension and macrosomia, were recorded and compared between high and low circVEGFC groups. It was observed that high expression levels of circVEGFC were correlated with fetal malformation and hypertension (Table 1), but not other adverse events. Besides, using Youden's index as the cutoff value for grouping, essentially the same results were obtained (data not shown). In contrast, GDM was closely correlated with all monitored

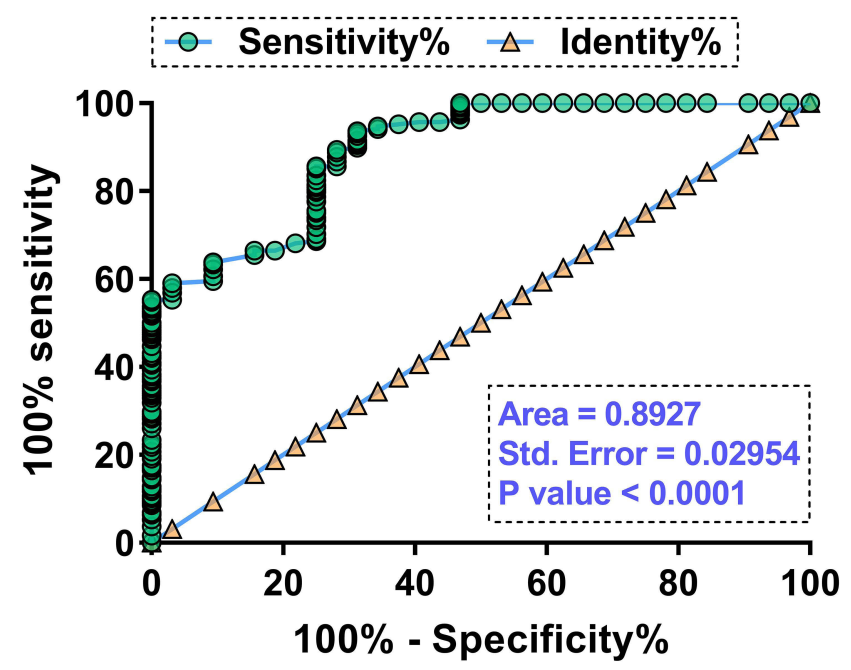

Figure 4 Plasma expression levels of circVEGFC provide high sensitivity and specificity in early diagnosis of GDM. With GDM patients as true positive cases and non-GDM patients as true negative cases, the early diagnostic value of plasma circVEGFC for GDM was analyzed by performing ROC curve analysis.

adverse effects, including malformation, hypertension, premature delivery, miscarriage, intrauterine distress, intrauterine death, intrauterine infection and macrosomia (Table 1).

\section{Discussion}

In this study, we explored the differential expression of circVEGFC in GDM and evaluated its potential in predicting GDM and its adverse complications. We showed that circVEGFC may serve as an early predictor of GDM.

CircVEGFC is a novel circRNA with critical functions in high glucose-induced cell injury. ${ }^{15}$ It was reported that 
Table I Comparisons of the Incidence Rates of Multiple Adverse Events Between High and Low circVEGFC Level Groups

\begin{tabular}{|c|c|c|c|c|c|}
\hline \multirow[t]{2}{*}{ Events } & \multirow[t]{2}{*}{ Cases } & \multicolumn{2}{|c|}{ CircVEGFC } & \multicolumn{2}{|c|}{ GDM } \\
\hline & & High $(n=|| 0)$ & Low $(n=|| 0)$ & Yes $(n=32)$ & No $(n=188)$ \\
\hline Malformation & 18 & $14 *$ & 4 & $12^{*}$ & 6 \\
\hline Hypertension & 52 & $38^{*}$ & 14 & $24 *$ & 28 \\
\hline Premature delivery & 12 & 7 & 5 & $\mathrm{II} *$ & 1 \\
\hline Miscarriage & 24 & 14 & 10 & $20 *$ & 4 \\
\hline Intrauterine distress & 61 & 34 & 27 & $25^{*}$ & 36 \\
\hline Intrauterine death & 5 & 3 & 2 & $4 *$ & 1 \\
\hline Intrauterine infection & 3 & 2 & I & $3 *$ & 0 \\
\hline Macrosomia & 11 & 6 & 5 & $10 *$ & 1 \\
\hline
\end{tabular}

Note: ${ }^{*} \mathrm{p}<0.05$

circVEGFC may interact with the miR-338-3p/HIF-1 $\alpha /$ VEGFA axis to promote the injuries of vascular endothelial cells induced by high glucose. ${ }^{15}$ It is well established that high glucose-induced cell injuries contribute to the development of GDM and its complications. ${ }^{16}$ Therefore, circVEGFC is likely involved in GDM. In this study, it reported increased expression levels of circVEGFC in GDM patients at multiple time points. Interestingly, the expression levels of circVEGFC were further increased after delivery. The possible explanation is that, besides pregnancy, circVEGFC is also involved in other physiological and pathological processes after delivery. Although certain biomarkers, such as $\mathrm{HbA1c}$, have been developed to predict GDN in the first trimester, the applications of these biomarkers are limited by the low sensitivity and specificity. ${ }^{17}$ In this study, increased expression levels of circVEGFC on the day of admission were observed in females who developed GDM during follow-up, and increased expression levels of circVEGFC on the day of admission effectively distinguished GDM patients from the controls. Moreover, pregnant women in high circVEGFC level group showed a significantly higher incidence of GDM compared to the ones in low circVEGFC level group. Therefore, detecting the expression levels of circVEGFC in plasma of pregnant women may assist the identification of pregnant women with high risk of GDM, thereby assisting the development of individualized preventative approaches. However, this study only observed 2 and 1 cases of suspected type 2 diabetes at 6 and 12 weeks after delivery, respectively. The role of circVEGFC as an early biomarker for type 2 diabetes remains to be further evaluated by future studies with a bigger sample size.

Interestingly, the present study observed that high expression levels of circVEGFC were correlated with fetal malformation and hypertension, but not other adverse events. Therefore, the main role of circVEGFC in GDM is likely involved in the development of adverse events in both fetus and mother. However, the present study did not include animal model experiments to investigate the role of circVEGFC in the development of GDM. Future studies may explore the role of circVEGFC in fetal malformation and hypertension using animal models. In addition, this study only enrolled 220 Han Chinese. Conclusions of the present study should be validated by future studies with more participants from different populations.

In conclusion, circVEGFC was upregulated in GDM. High expression levels of circVEGFC may serve as an early predictor of GDM and its adverse events.

\section{Abbreviation}

GDM, gestational diabetes mellitus.

\section{Disclosure}

All the authors have declared that in the research there was no potential conflict of interest.

\section{References}

1. McIntyre HD, Catalano P, Zhang C, Desoye G, Mathiesen ER, Damm P. Gestational diabetes mellitus. Nat Rev Dis Primers. 2019;5(1):47.

2. Szmuilowicz ED, Josefson JL, Metzger BE. Gestational diabetes mellitus. Endocrinol Metab Clin North Am. 2019;48(3):479-493. doi:10.1016/j.ecl.2019.05.001

3. Johns EC, Denison FC, Norman JE, Reynolds RM. Gestational diabetes mellitus: mechanisms, treatment, and complications. Trends Endocrinol Metab. 2018;29(11):743-754. doi:10.1016/j. tem.2018.09.004

4. Nguyen CL, Pham NM, Binns CW, Duong DV, Lee AH. Prevalence of gestational diabetes mellitus in Eastern and Southeastern Asia: a systematic review and meta-analysis. $J$ Diabetes Res. 2018;2018:6536974. doi:10.1155/2018/6536974

5. Chiefari E, Arcidiacono B, Foti D, Brunetti A. Gestational diabetes mellitus: an updated overview. J Endocrinol Invest. 2017;40(9):899909. doi:10.1007/s40618-016-0607-5 
6. Xu T, Dainelli L, Yu K, et al. The short-term health and economic burden of gestational diabetes mellitus in China: a modelling study. BMJ Open. 2017;7(12):e018893. doi:10.1136/bmjopen-2017-018893

7. Farrar D, Simmonds M, Bryant M, et al. Treatments for gestational diabetes: a systematic review and meta-analysis. BMJ Open. 2017;7 (6):e015557. doi:10.1136/bmjopen-2016-015557

8. Njete HI, John B, Mlay P, Mahande MJ, Msuya SE. Prevalence, predictors and challenges of gestational diabetes mellitus screening among pregnant women in northern Tanzania. Trop Med Int Health. 2018;23(2):236-242. doi:10.1111/tmi.13018

9. Immanuel J, Simmons D. Screening and treatment for early-onset gestational diabetes mellitus: a systematic review and meta-analysis. Curr Diab Rep. 2017;17(11):115. doi:10.1007/s11892-017-0943-7

10. Powe CE. Early pregnancy biochemical predictors of gestational diabetes mellitus. Curr Diab Rep. 2017;17(2):12. doi:10.1007/ s11892-017-0834-y

11. Sweeting AN, Wong J, Appelblom H, et al. A novel early pregnancy risk prediction model for gestational diabetes mellitus. Fetal Diagn Ther. 2019;45(2):76-84. doi:10.1159/000486853

12. Yan Q, He X, Kuang G, Ou C. CircRNA cPWWP2A: an emerging player in diabetes mellitus. J Cell Commun Signal. 2020;14(3):351353. doi:10.1007/s12079-020-00570-7
13. Yan L, Feng J, Cheng F, et al. Circular RNA expression profiles in placental villi from women with gestational diabetes mellitus. Biochem Biophys Res Commun. 2018;498(4):743-750. doi:10.1016/ j.bbrc.2018.03.051

14. Wang H, She G, Zhou W, Liu K, Miao J, Yu B. Expression profile of circular RNAs in placentas of women with gestational diabetes mellitus. Endocr J. 2019;66(5):431-441. doi:10.1507/endocrj.EJ18-0291

15. Wei H, Cao C, Wei X, et al. Circular RNA circVEGFC accelerates high glucose-induced vascular endothelial cells apoptosis through miR-338-3p/HIF-1 $\alpha /$ VEGFA axis. Aging. 2020;12(14):1436514375. doi:10.18632/aging.103478

16. Peng HY, Li HP, Li MQ. High glucose induces dysfunction of human umbilical vein endothelial cells by upregulating miR-137 in gestational diabetes mellitus. Microvasc Res. 2018;118:90-100. doi:10.1016/j.mvr.2018.03.002

17. Benaiges D, Flores-le Roux JA, Marcelo I, et al. Is first-trimester HbA1c useful in the diagnosis of gestational diabetes? Diabetes Res Clin Pract. 2017;133:85-91. doi:10.1016/j.diabres.2017.08.019

\section{Publish your work in this journal}

Diabetes, Metabolic Syndrome and Obesity: Targets and Therapy is an international, peer-reviewed open-access journal committed to the rapid publication of the latest laboratory and clinical findings in the fields of diabetes, metabolic syndrome and obesity research. Original research, review, case reports, hypothesis formation, expert opinion and commentaries are all considered for publication. The manuscript management system is completely online and includes a very quick and fair peer-review system, which is all easy to use. Visit http://www.dovepress.com/testimonials.php to read real quotes from published authors. 